\title{
The Disk Covering Tour Problem in the Wireless Sensor Network
}

\author{
Li Chunli* and Mu Ke
}

\author{
School of Mathematics and Statistics, Zhoukou Normal University, Zhoukou, 466000, China
}

\begin{abstract}
The paper discusses the disk covering tour problem for reducing the mobile devices' mobile electricity costs consumed in the wireless sensor network. The DCTP discusses how to find a tour whose cost is the minimum under the condition of the defined initial points and some planar points. The tour starts from the initial stop point. After passing many stop points, the tour returns to the initial stop point. Although the stop point is unnecessary to be in the defined points, the distance between each defined point and a certain stop point during the tour at least should be within the defined disk radius. The electricity can be consumed much when the mobile devices move in and out the stop points. Therefore, the less the number of the stop points is, the less the tour cost is. How to reduce the number of the stop points is regarded as the disk covering problem. The decreasing k-means algorithm is proposed to try finding out the nearly minimum disk for covering all defined points, and then the Lin-Kernighan heuristic is introduced to find out the approximating shortest tour through the obtained stop points. The experimental simulations compare solution algorithms between the method proposed in the paper and other related methods with the Qi-Ferry method for finding out the shortest tour, and then the results show that the method proposed in the paper has the advantage of having a smaller tour cost.
\end{abstract}

Keywords: Covering tour problem, disk covering problem, K-means algorithm, wireless sensor network.

\section{INTRODUCTION}

With more and more mature mobile devices, many issues have conducted different applications by the use of the mobile devices to the wireless sensor network, such as the data collection, the use of the mobile sink and others. Discussing how to effectively control the electricity consumption in the mobile devices is an important issue because the mobile devices' functions and its applied environments are very different and its operating ability is just limited in the electricity itself [1].

The electricity consumption in the mobile devices consists of the service provided by the mobile devices and its mobile electricity consumption. The former means the service provided by the mobile devices to the sensors, such as wireless charging, data collection and others [2]. The later means the mobile electricity consumption during the process of providing the service by the mobile devices. It is called as the tour cost in the paper. The tour cost means the electricity consumed by the mobile devices leave or enter the stop point with acceleration and deceleration and pass the neighboring stop point between the two tours with the constant speed. In general, the number of the stop points can the length of the tour can influence the tour cost [3]. The paper mainly discuss how to reduce mobile devices' tour cost and its electricity consumption, that is, the mobile devices' mobile electricity consumption can be reduced by choosing the proper deformation traveling salesman problem [4-6].
The DCTP is introduced in the paper and defined as to find a tour whose cost is the minimum under the condition of the defined initial points and some planar points. The tour starts from the initial stop point. After passing many stop points, the tour returns to the initial stop point. Although the stop point is unnecessary to be in the defined points, the distance between each defined point and a certain stop point during the tour at least should be within the defined disk radius. The problem conforms with some wireless sensor networks, such as the data collection with the use of the mobile data mule, the wireless charging with the use of the wireless mobile charger and others. Its main purpose is to reduce mobile devices' tour cost.

There is a trend that the less the number of the stop points is, the less the tour cost is. The known algorithms have been discussed that many methods can be adopted to reduce the problem of the number of the stop points, such as Set Covering Problem, Disk Covering Problem, Partial Covering Problem and others, in which a large part of methods regard the nodes as the candidates of the stop points. Therefore, the number of the stop points is not optimal, as shown in the Fig. (1). Compared with the number of the stop points in other positions (on the right), the number of the stop points in the nodes (on the left) is more. Reducing the number problem of the stop points is regarded as the DCTP. The fixed information requires to be defined in advance when the previous SCP method and DCP method are solved, such as relation set, circle set and others, the higher algorithm complexity will be caused. The DCP can cover a node and a circle whose radius is $r$ which just has no more than $4 \pi r 2$. If all circles caused by all nodes are discussed, the computing cost will be very high. 

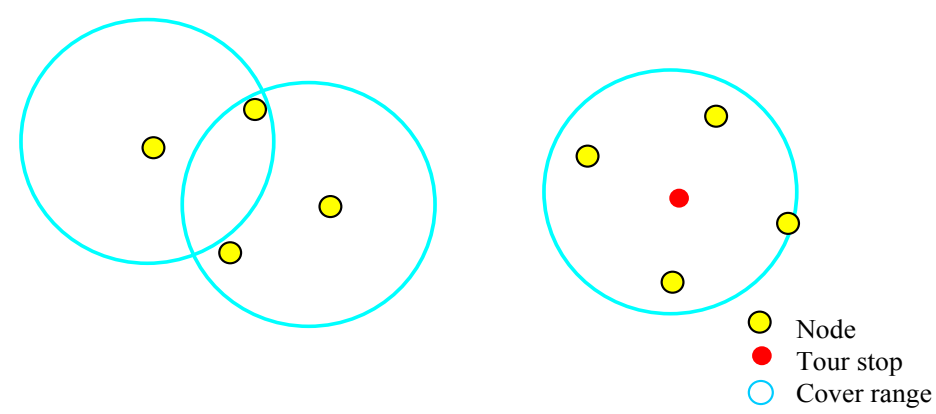

Fig. (1). The position covering example in the stop points,

The Decreasing K-means Algorithm is introduced to solve the DCTP, and the Greedy Algorithm is proposed in the reference [7] and modified into the complete covering problem. The obtained number of the circles is regarded as the clustering number of the initial K-means. The K-means is conducted as the disk covering problem and then the clustering relationship can be obtained. Later, the Smallest Enclosing Circle Problem can be discussed to modify the original clustering center and be regarded as the stop point. The approximating shortest tour can be found out through the LKH algorithm. In addition, the different tours can be obtained with the iteration and all points covered by the approximating minimum disk can be found out with the deceleration. A proper solution can be selected in each round to find out a smaller tour cost. Although reducing the number of the stop points can increase the length of the tour or the tour cost, the simulative results show that there is a tendency that the the length of the tour or the tour cost obtained by the DK-means can be reduced when the number of the stop points is fewer. What's more, another advantage of the DK-means can choose a smaller tour cost, though its obtained number of the stop points is not the smallest. The method proposed in the paper can possess the advantage of a smaller tour cost by simulating DK-means and comparing with Qi-Ferry and CSP. Therefore, the more the defined numbers are, the more obvious the advantages of the method is.

\section{RELATED RESEARCHES}

The is one of the most famous combination optimal problems nowadays. In brief, the shortest tour should be found out under the defined known points. It starts from a node and then returns to the initial node after visiting other each node. In recent years, many newly deformed Traveling Salesman Problem has been constantly introduced in the research, such as Clustered Traveling Salesman Problems, Traveling Salesman Problems with Profits and others.

A heuristic method proposed in the paper [3] to solve the CSP, and the CSP is a kind of the deformed Traveling Salesman Problem. Its definition is that each point $p$ has the previous defined covering distance $\mathrm{dp}$ in the defined $\mathrm{n}$ points and a sub set can be found out $\mathrm{n}$ the defined $\mathrm{n}$ points. Later, the points in the sub set are regarded as the stop points and then form a tour whose cost is the minimum. The distance between each point $i$ which does not belong to the stop point and a stop point should be at least within di. The problem can be used in the real world, such as the visiting behavior of the routing rural medical\&health service team. It assumes that it is unnecessary to visit each city for the service team just can set up the stop station in some cities and those people who live in the city where there are no stop stations just needs to move into the nearest stop station. The author firstly finds out all solutions in the Set Covering Problem and then choose the shortest tour in the tours formed by all solutions. The method proposed in the paper is called as CSP.

The reference [4] in the paper proposes that the environment can help charge the sensors (nodes) in the wireless sensor network through the wireless mobile chargers, in which the author adopts to the covering concept in the CSP. The stop point is not in the node position so that finding out a stop point combination can make the tour be the shortest and the mobile chargers' electricity consumption in the wireless sensor network can be reduced. Its final purpose is to increase the number of the charged sensors. The heuristic method proposed by the author can obtain the tour whose solution is approximating the optimal solution through the particle clustering algorithm determines the stop point position and the LKH [5] should be matched. The optimal tour route can be found out by constantly modifying the stop point position. In addition, the length of the tour can be compared between the simulative method and the CSP, and it is called Qi-Ferry in the paper. The extra electricity consumption can be caused without considering the number of the stop point position. The reference [6] shows that the electricity when the mobile devices starts from the static situation to the acceleration or the constant speed and from the constant speed or the deceleration to the static situation will be consumed more than the electricity consumed in the fixed speed. The more the number of the stop points during the tour has, the more the electricity consumed in these behaviors. The last chapter in the paper can consider the data in the reference [6].

The reference [8] introduces the greedy algorithm with the use of the reference [7] as the Partial Covering Problem. The circle set whose radius is $r$ is caused through all random two nodes in the paper and the circles which have the most covering nodes can be selected again in these circle sets with the use of the greedy selected method, and then the number of the circles which is existed in advance can be obtained. 


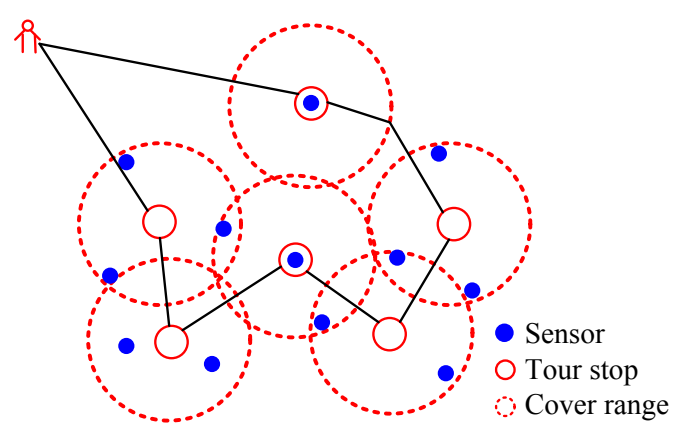

Fig. (2). DCTP application context.

In this way, the node covering rate in the network can be increased and the advantage of its method is simple. What's more, the circle is caused by the two different nodes so that the number of the candidate circles can be largely reduced. The author in the paper introduces some algorithms to discuss the partial covering problems, including Dominating Set (DS), Connectivity Algorithm (CA), and those three methods in the reference [7]. The results show that the greedy algorithm in the reference [7] is the optimal method in the covering rate results. Therefore, the greedy algorithm proposed in the reference [7] should be modified properly and makes all nodes be covered in the needed number of the circles. In addition, its values are used as the important parameters of the algorithm.

\section{RELATED RESEARCHES THE PROBLEM DEFI- NITION AND THE APLLICATION CONTEXT}

\subsection{Disk Covering Tour Problem, DCTP}

The following definition is the definition of the DCTP, in which $\mathrm{G}=(\mathrm{V}, \mathrm{E})$ is a complete figure in the Euclidean space and its set of the vertices is $V=\{d\} \cup Z$, in which $d$ represents the initial position and $Z$ represents the covered point sets. The non-negative function $C(i, j)$ is the Euclidean distance between the random two positions in the Euclidean space, and its purpose is to find a (Tour) $\mathrm{T}=(\mathrm{t} 1, \mathrm{t} 2, \cdots \mathrm{t}|\mathrm{T}|)$ whose cost is the minimum, in which ti is called Tour Stop and the non-negative $\mathrm{X}(\mathrm{i})$ is the cost of the stop point $i$.

1. The position of each stop point $t_{i} \in T$ is different, and $\mathrm{d}$ represents the initial and ending position, that is, $t_{i}=t_{(|T|+1)}=d$.

2. The distance between all nodes in the point set $\mathrm{Z}$ and a stop point tk should be at least smaller than the defined covering distance $r$.

The purpose function is as shown in the formula, in which $\mathrm{w}$ is the cost weighted value of the tour length.

Minimize $Z=w^{*} \sum_{i=1}^{|T|} C\left(t_{i}, t_{i+1}\right)+\sum_{i=1}^{|T|} X(i)$

\subsection{The Application of the Wireless Sensor Context}

DCTP is applied in the issue of using many mobile devices in the wireless sensor context nowadays, as shown in the Fig. (2). The deployment method adopts to the random deployment and it is moved to the stop position through the mobile devices. In this way, the sensors must be within the covering range operated in the stop point and then the proper tour should be selected to reduce the mobile devices' tour costs during the process of its operation, in which the function cost in the stop point is the consumed electricity when the mobile devices enter or leave the stop points with the acceleration and deceleration and the electricity consumed in the neighboring stop points between the two tours with the constant speed (the weighted value $\mathrm{w}$ is the consumed electricity whose mobile devices move one meter with the constant speed). The applied issues are as follows:

Wireless charging: the mobile charging device can help sensors charge with the fixed charging distance $r$ in the QiFerry wireless charging environment. The author assumes that the sensors lie in the charging range of the mobile charging devices and the received charging efficiency is the same, while the actual charging technology efficiency is inversely proportional to the distance square, such as Radio Frequency, RF. Therefore, the charging electricity efficiency obtained from the sensors within the charging range can require the meet of itself.(the charging efficiency is different from the charging technology.)

Data mule: the node information in the wireless sensor network can be collected through the mobile devices, and its wireless communication distance is the covering range.

\section{DECREASING K-MEASNS METHOD}

There is a trend that the smaller the number of the stop points is, the smaller the tour costs cause. Therefore, the minimum number of the stop points can be found out with the use of K-means clustering algorithm and the diminishing concept. Each clustering relationship obtained from the Kmeans adopts the concept of the Smallest Enclosing Circle Problem to try whether the disk which is smaller than the size of the covering range can cover other clustering members, and the obtained center in the smallest enclosing circle is the stop position. In addition, the approximating shortest tour can be found out through the obtained stop points and Lin-Kernighan Heuristic. What's more, the iterative deceleration method can be adopted to increase tours formed by many different stop point combinations.

The Fig. (3) is the proposed DK-means method algorithm, in which $\mathrm{S}$ is the point set and $\mathrm{R}$ is the covering 


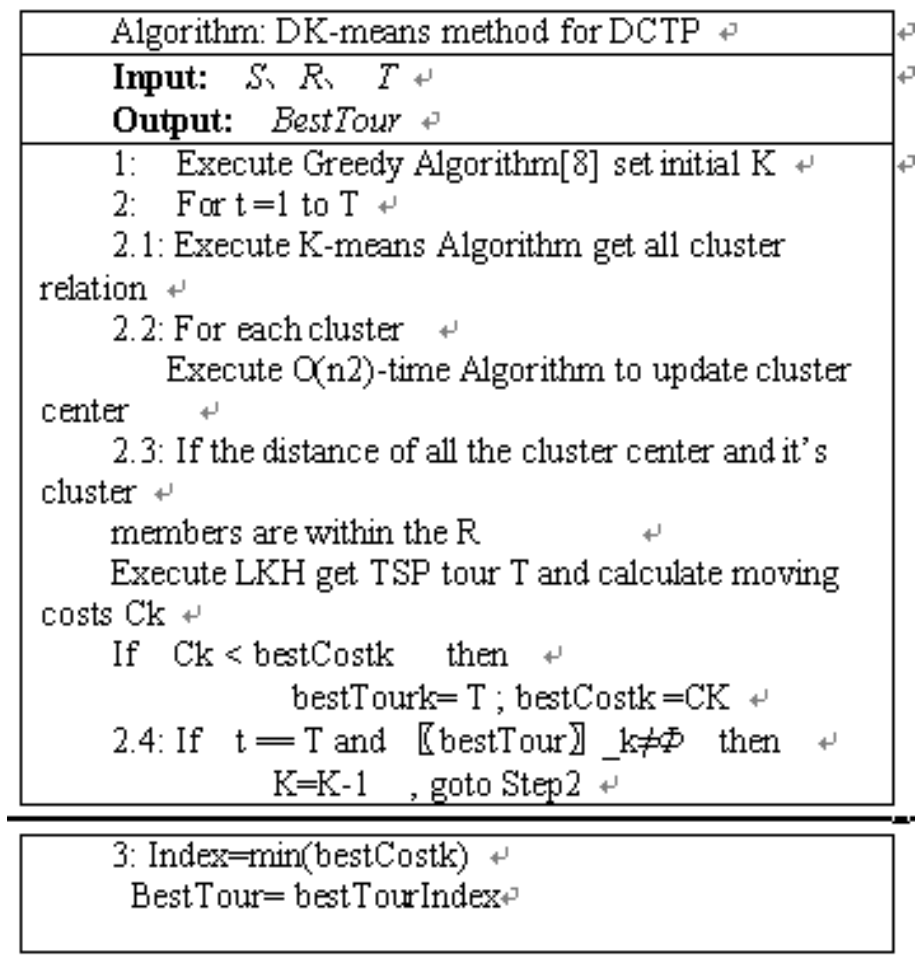

Fig. (3). DK-means method for DCTP.

distance. $\mathrm{T}$ is the circular operation times and is set up in terms of observing the experimental results. Step 1: K value can be obtained with the operation of the Greedy Algorithm and then the $K$ value can be used as the feasible solution so that a lower $\mathrm{K}$ value can begins to find out a proper covering result. Step 2: the tours formed by many different stop point combinations can be obtained with the iteration method. When the K-means Algorithm is operated in each round, the clustering relationship can be obtained, and each clustering relationship can respectively regard the center obtained from the smallest enclosing concept (refer to the $\mathrm{O}(\mathrm{n} 2)$-time Algorithm [9] solution) as the newly clustering center. If the modified clustering center can cover all nodes, the clustering center can be regarded as the stop point and the tour information can be obtained by operating LKH algorithm. Moreover, the tour costs can be computed. If it is smaller than the regional optimal results during the comparison of the regional optimal results, the regional optimal result can be replaced. When the circular times operate to the defined times $\mathrm{T}$ and there is the regional optimal solution ( $\mathrm{K}$ disks can be used to cover all nodes), $\mathrm{K}$ should be minus 1 , and then it should be returned to the step 2 for checking whether there is a smaller $\mathrm{K}$ value. Otherwise the Step 3 should be operated. Step 3: the tour route whose tour costs are the smallest in all regional optimal results, and making the BestTour represent the final result.

\section{EXPERIMENTAL SIMULATION}

\subsection{Simulational Environment}

The Matlab is adopted to as the simulational tools, and its method is applied in the wireless sensor network in the mobile devices. The different numbers of the sensors from 30 to 120 should be randomly deployed in the $100 \mathrm{~m} \times 100 \mathrm{~m}$ simulational context and its corresponding operating times is $3000,5000,8000$ and 10000 . The length of the disk covering radius is $10 \mathrm{~m}$. In addition, $\mathrm{K}$-means selects the initial clustering center with the random methods and the initial position in the mobile devices is set up in the left corner. The initial position can be regarded as the base station or other devices, so the sensors next to the initial position can be served by the mobile devices in the first time. The mobile devices adopt the algorithms to determine the tour routes and serve the nodes in the wireless sensor network properly.

The mobile behaviors in the mobile devices is adopted to weigh the cost of the stop point. In order to actually estimate the extra electricity consumed in the mobile devices from the acceleration, deceleration to the fixed speed, the actual tested data in the reference [6] is used as basis. According to the formulas provided in the paper and the data derived from the results of the Fig. (4), and the reference $w$ in the figure is the angle speed of the motor which is set as the result of 3600 $\mathrm{rad} / \mathrm{s}$, the horizontal axis coordinate is the moving distance in the present mobile devices and the vertical axis coordinate is the electricity consumption per meter. The figure shows that the extra electricity consumption and the distance is two meters during the process of changing the mobile devices' speed from the static or accelerated speed to the constant speed. In addition, the electricity consumed in the accelerated speed and the decelerated speed is the same. However, the electricity consumption between the two points maybe smaller than the distance sum whose speed is accelerated to the fixed speed and the fixed speed is decelerated to the static situation. Therefore, the distance between the two stop 


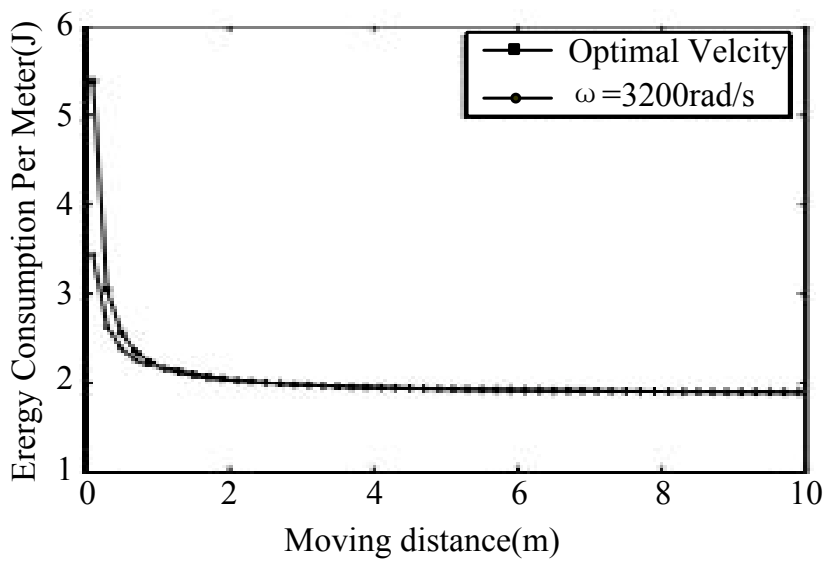

Fig. (4). The electricity consumption relation when the mobile devices' static situation accelerated to the fixed speed.

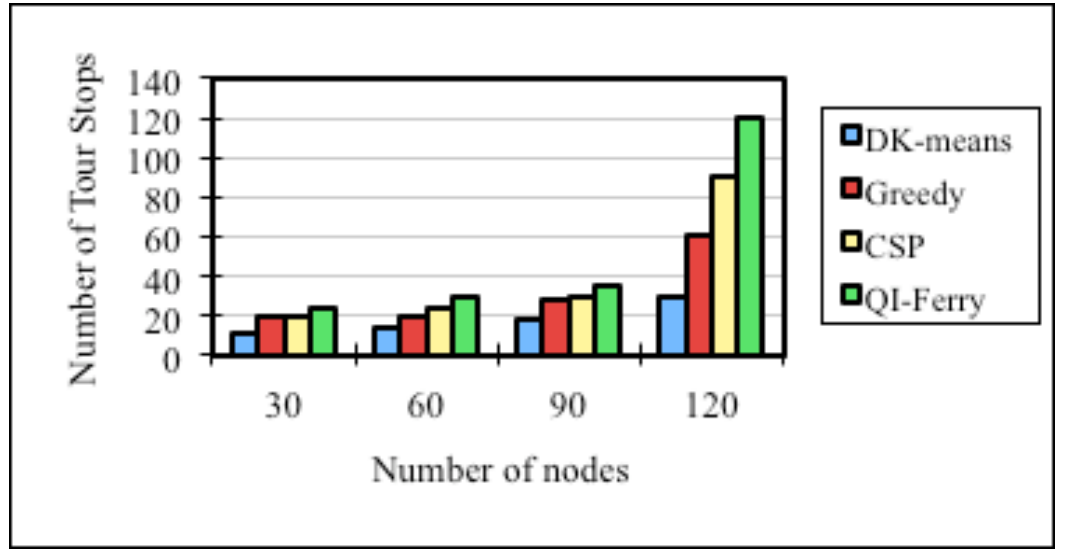

Fig. (5). The comparison of the number of the stop points among different algorithms.

points should be put in the formulas and the parameters in the references when the extra electricity consumption between the stop points should be computed.

\subsection{Efficiency Analysis}

In order to estimate its efficiency, the mainly considered three kinds of measurements are the number of the stop point, the length of the tour and the tour cost. Although the paper regards the tour cost as the purpose in the algorithm Fig. (3), the electricity consumed in the stop points is enough small to be ignored, the algorithm regards the length of the tour and the tour cost as the two kinds of the purpose orientation, that is, the condition of judging the regional optimal solution in the Step 2-3 of the original algorithm can be changed with the different measuring standards.(the tour cost should be considered originally).

\subsection{Efficiency Estimation}

Compared with the Greedy [8], the method obtained from the Fig. (5) has fewer number of the stop points for the number of the stop points obtained from the Greedy method is decreasing. The approximating minimum disk should be found out to cover all nodes and the stop points in the CSP just can be selected in the nodes so that there are more numbers of the stop points. Each node position in the Qi-Ferry can choose a stop point so that the number of the stop point is the same to the number of the node.

When the number of the network node is 30 and 60 in the Fig. (6), the length of the tour in the Qi-Ferry is more than others' tour length for its tour route is obtained by adopting the particle clustering algorithm to modify it in the Qi-Ferry. When the number of the network node is 90 and 120, the QiFerry adopts the basic particle clustering algorithm to restrain the disadvantages of the regional optimal solution so that its length of the tour will be slowly decreased compared with other methods, while CSP possesses the selected problem for the tour route so that its effect is worse than that of the new method. When the number of the node is 30 in the Fig. (7), the tour cost in the new method is more than Qi-Ferry's for its number of the nodes is very little. What's worse, the advantages of reducing the number of the stop points is not obvious. With the increasing of the number of the nodes, the new method's tour cost is better than that of Qi-Ferry and CSP. It proves that the electricity consumption of the tour cost can be reduced by reducing the number of the stop points. 


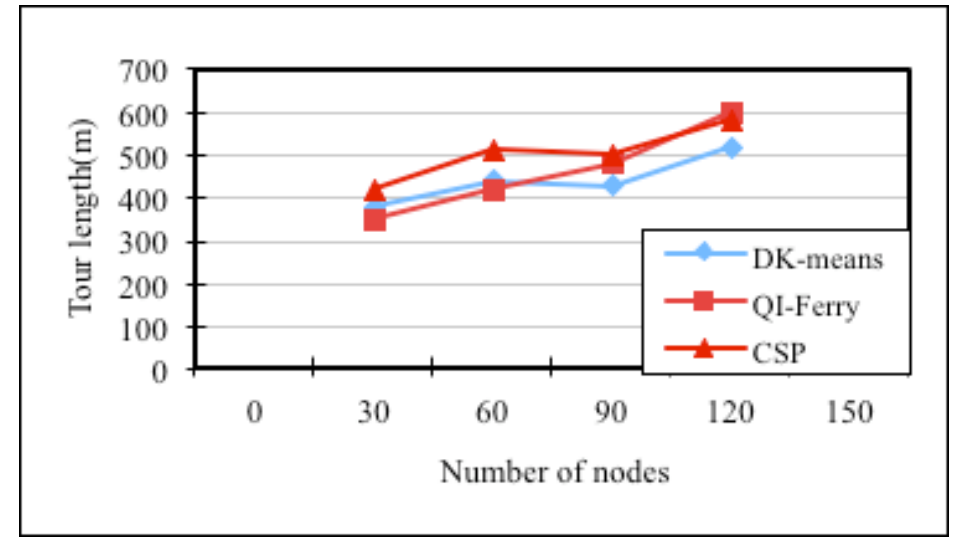

Fig. (6). The comparison of the tour length among different network node numbers in the mobile devices.

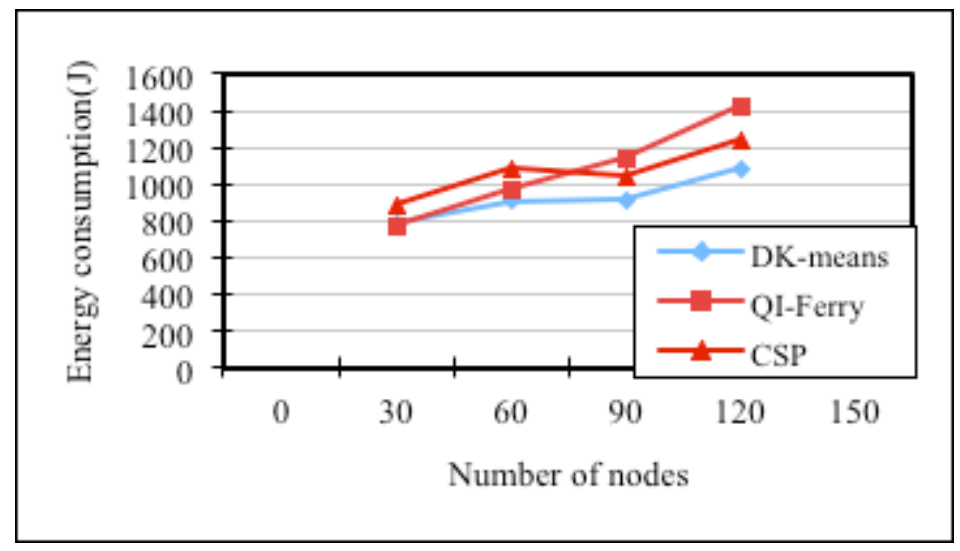

Fig. (7). The comparison of the tour cost orientation among different network node numbers in the mobile devices.

\section{CONCLUSION}

The DCTP is introduced in the paper and applied in the wireless sensor network context. The method is different from the Covering Salesman Problem and the Qi-Ferry Problem. The stop point in the Covering Salesman Problem requires to be selected from the node positions and the QiFerry Problem does not consider the non-ignorable electricity consumtion caused by the number of the stop points to the mobile devices. The DK-means method is proposed to obtain a fewer tour length and the tour cost by finding out the approximating minimum number of the stop points. Compared with the CSP and the Qi-Ferry, the paper shows that the proposed method has the advantage of having a fewer tour cost. If the more the number of the network nodes has, the more obvious the advantage of the DK-means efficiency compared with other two kinds of methods.

The number of the optimal stop point and the position of the optimal stop point should be obtained through the improved method in the future job. Later, the fewer tour costs should be computed by modifying the optimal stop point positions with the use of the proper algprithms, such as genetic algorithm, ant colony algorithm and others. The method is discussed in the paper with the use of K-means and the smallest enclosing modifying clustering center and the parameters are modified to improve the partial covering problem.

\section{CONFLICT OF INTEREST}

The authors confirm that this article content has no conflict of interest.

\section{ACKNOWLEDGEMENTS}

This work is supported by the Key Project of Guangxi Social Sciences, China (No.gxsk201424), the Education Science fund of the Education Department of Guangxi, China (No.2014JGA268), and Guangxi Office for Education Sciences Planning, China (No.2013C108).

\section{REFERENCES}

[1] T.S. Chen, and P.W. Wu, "On data collection using mobile robot in wireless sensor networks," IEEE Transactions on Systems, Man and Cybernetics, vol. 41, pp. 1213-1224, 2011.

[2] M. Zungeru, L.M. Ang, and K.P. Seng, "Termite-hill: routing towards a mobile sink for improving network lifetime in wireless sensor networks," In: $3^{\text {rd }}$ International Conference on Intelligent Systems, Modelling and Simulation (ISMS), 2012, pp. 622-627.

[3] J. Current, and D. Schilling, "The covering salesman problem," Transportation Sciences, vol. 23, pp. 208-213, 1989.

[4] K. Li, H. Luan, and C.C. Shen, "Qi-Ferry: energy-constrained wireless charging in wireless sensor networks," In: IEEE Wireless Communications and Networking Conference, 2012, pp. 25152520 . 
[5] K. Helsgaun, "An effective implementation of the Lin-Kernighan traveling salesman heuristic," European Journal of Operational Research, vol. 126, no. 1, pp. 106-130, 2000.

[6] G. Wang, M.J. Irwin, P. Berman, H. Fu, and T.L. Porta, "Optimizing sensor movement planning for energy efficiency," In: Proceedings of the International Symposium on Low Power Electronics and Design, 2005, pp. 215-220.

[7] B. Xiao, J. Cao, Q. Zhuge, Y. He, and E.H.M. Sha, "Approximation algorithms design for disk partial covering problem," In: Pro- ceedings in $7^{\text {th }}$ International Symposium on Parallel Architectures, Algorithms and Networks, 2004, pp.104-109.

[8] L.W. Chen, and C.C. Chen, "The partial coverage problems in wireless sensor networks," The $4^{\text {th }}$ Workshop on Wireless Ad Hoc and Sensor Networks, pp. 95-102, 2008.

[9] J. Elzinga, and D.W. Hearn, "The minimum covering sphere problem," Management Science, vol. 19, pp. 96-104, 1972.

(C) Chunli and Ke; Licensee Bentham Open.

This is an open access article licensed under the terms of the Creative Commons Attribution Non-Commercial License (http://creativecommons.org/licenses/by$\mathrm{nc} / 3.0 /$ ) which permits unrestricted, non-commercial use, distribution and reproduction in any medium, provided the work is properly cited. 